Check for updates

Cite this: J. Mater. Chem. A, 2017, 5 , 24651

Received 25th August 2017

Accepted 10th November 2017

DOI: $10.1039 / c 7 t a 07495 f$

rsc.li/materials-a

\section{D hierarchical porous structured carbon nanotube aerogel-supported Sn spheroidal particles: an efficient and selective catalyst for electrochemical reduction of $\mathrm{CO}_{2}$ to formate $\uparrow$}

\author{
Zhipeng Chen, ab Shunyu Yao a and Licheng Liu (DD *a
}

A 3D hierarchical porous structured carbon nanotube aerogelsupported Sn spheroidal particles (Sn/CNT-Agls) on carbon cloth (Sn/CNT-Agls/CC) electrode as a selective and efficient cathode for electrochemical reduction of $\mathrm{CO}_{2}$ to formate is reported. The electrocatalytic activity of $\mathrm{Sn} / \mathrm{CNT}$-Agls/CC toward $\mathrm{CO}_{2}$ reduction is greatly enhanced in comparison with carbon nanotube-supported $\mathrm{Sn}$ spheroidal particles (Sn/CNT) on carbon cloth (Sn/CNT/CC) electrodes, which is attributed to the high specific surface area, superior conductivity and excellent 3D hierarchical structure of Sn/CNT-Agls. A maximum faradaic efficiency of $82.7 \%$ for formate production has been achieved with high stability at $-0.96 \mathrm{~V}$. Strikingly, the selective $\mathrm{CO}_{2}$ reduction to formate can occur at a low overpotential of $361 \mathrm{mV}$, and a large current density of $32.9 \mathrm{~mA} \mathrm{~cm}{ }^{-2}$ is obtained on the Sn/CNT-Agls/CC electrode.

The conversion of $\mathrm{CO}_{2}$ to useful carbon-based fuels is an attractive way to deal with rising $\mathrm{CO}_{2}$ emission. ${ }^{1}$ Recently, the electrochemical reduction of $\mathrm{CO}_{2}$ for producing value-added fuels in aqueous solutions has attracted much attention due to its high selectivity and energy-efficiency. ${ }^{2-5}$ Among the various products of electrochemical reduction of $\mathrm{CO}_{2}$, formate is one of the most important products with a high economic value. ${ }^{6}$ Formate is a basic organic chemical raw material, which is widely used in leather, dye, pharmaceutical and rubber industries, and it can also be directly used in fuel cells to generate electricity. ${ }^{7-10}$ Thus, it is very interesting to develop an electrocatalyst that can reduce $\mathrm{CO}_{2}$ to value-added formate with high electrocatalytic activity.

To date, a great number of metal or metal oxide electrocatalysts have been used in the electrochemical reduction of $\mathrm{CO}_{2}$ for producing formate. ${ }^{11-15}$ Among these various metal

${ }^{a}$ CAS Key Laboratory of Bio-based Materials, Qingdao Institute of Bioenergy and Bioprocess Technology, Chinese Academy of Sciences, Qingdao 266101, Shandong, China. E-mail: liulc@qibebt.ac.cn; Tel: +86-532-80662780

${ }^{b}$ University of Chinese Academy of Sciences, Beijing 100049, China

$\dagger$ Electronic supplementary information (ESI) available. See DOI: $10.1039 / \mathrm{c} 7 \mathrm{ta} 07495 \mathrm{f}$ electrodes, Sn-based electrodes are widely investigated because of their cost-effectiveness and nontoxicity as well as high selectivity to formate. ${ }^{16-20}$ Although Sn electrodes exhibit high faradaic efficiency toward $\mathrm{CO}_{2}$ reduction, a poor current density and production rate are observed during the process of electrolysis. For example, Zhang et al. reported an ultrafine nanostructured Sn catalyst and achieved a high faradaic efficiency of $\sim 93 \%$ for producing formate with a current density of $\sim 5.4 \mathrm{~mA} \mathrm{~cm}^{-2}$ at $-1.8 \mathrm{~V}^{19} \mathrm{Lv}$ et al. explored the performance of $\mathrm{CO}_{2}$ reduction to formate on a foil electrode and obtained the highest faradaic efficiency of $\sim 91 \%$, but a current density of only $\sim 2.5 \mathrm{~mA} \mathrm{~cm}{ }^{-2}$ was observed. ${ }^{21}$ Furthermore, the electrocatalytic activity toward $\mathrm{CO}_{2}$ reduction exhibits a strong dependence on the structure and morphology of the electrocatalysts. As previously reported, the faradaic efficiency for formate production is shown in a wide range from 18\% to $93 \%$ over different Sn-based electrocatalysts. ${ }^{19,22-25}$ Therefore, developing a new morphological and structured Sn catalyst with a high current density, production rate and selectivity is still challenging and significant.

Carbon nanotube aerogel is a new kind of three-dimensional (3D) porous material with great potential in many fields because of its high specific surface area, good mechanical stability and superior conductivity. ${ }^{26,27}$ Although great progress has been made in carbon nanotube aerogel materials, there are no reports about metals or metal oxides supported on 3D porous carbon nanotube aerogels as electrocatalysts for the electrochemical reduction of $\mathrm{CO}_{2}$. Such a unique structure of the electrocatalyst is very attractive, as good conductivity and multidimensional mass transfer pathways can be provided.

Herein, a 3D hierarchical porous structured Sn/CNT-Agls electrocatalyst was fabricated via a simple three-step process. The biggest difference from previous studies about Sn electrodes is that our study provides a hierarchical porous structured carrier for Sn electrocatalysts. This unique structure of the electrocatalyst leads to a significant increase of current density toward $\mathrm{CO}_{2}$ reduction and the reasons can be attributed to the following two points. Firstly, the 3D porous structured CNT-Agls 
catalyst carrier can provide better mass transfer pathways for the $\mathrm{CO}_{2}$ reduction reaction. Therefore, the reactants $\left(\mathrm{CO}_{2}\right)$ can be more easily exposed to catalytically active sites ( $\mathrm{Sn}$ ). Secondly, the 3D porous structured CNT-Agls catalyst carrier has excellent conductivity, which can greatly accelerate the electron transfer rate during the reaction. We found that the asprepared Sn/CNT-Agls/CC electrode exhibited excellent catalytic performance toward $\mathrm{CO}_{2}$ reduction, including a large current density, a high production rate and selectivity, a good stability and a moderate overpotential. In order to evaluate the effect of the 3D hierarchical porous structure, the electrochemical and catalytic activities of 3D Sn/CNT-Agls and 2D Sn/CNT catalysts were compared.

The 3D structured Sn/CNT-Agls catalyst was fabricated by a three-step process as illustrated in Fig. 1 (see the Experimental section in the ESI $\dagger$ for details). Briefly, a stable hybrid aqueous suspension of CNTs, chitosan (CS) and $\mathrm{SnCl}_{4}$ was obtained by strong ultrasonic mixing and physical crosslinking (the CS played a role of the physical crosslinking agent in this experiment). Subsequently, the as-prepared hybrid suspension was frozen to ice and then it was freeze-dried to form $\mathrm{Sn}(\mathrm{OH})_{x} / \mathrm{CNT}$ Agls. Through the physical crosslinking and ice-template selfassembly method, the $\mathrm{Sn}^{4+}$ could be uniformly dispersed on the surface of the CNTs and then nucleated and grew on the CNT aerogel-skeleton. Finally, the as-prepared $\operatorname{Sn}(\mathrm{OH})_{x} / \mathrm{CNT}$ Agls was converted to Sn/CNT-Agls via a simple calcination reduction process under an $\mathrm{H}_{2}$ atmosphere. The weighing result reveals that the density of the as-prepared Sn/CNT-Agls is only $16.2 \mathrm{mg} \mathrm{cm}^{3}$.

The morphology and microstructure of the as-prepared $\mathrm{Sn} /$ CNT-Agls and Sn/CNT were characterized by SEM, TEM and BET. As shown in Fig. 2a and b, the 3D structured CNT-aerogels with mesopores and macropores were successfully prepared, and the micron-sized spheroidal Sn particles nucleated in the skeleton of the CNT-aerogels. The TEM results (Fig. S3†) show that the Sn particles are not evenly dispersed in the CNTaerogels, and the average size of the $\mathrm{Sn}$ particles is $c a$. $1.65 \mu \mathrm{m}$. But, the hierarchical porous 3D structured material was not observed in the Sn/CNT composite (Fig. S1a and c $\dagger$ ). $\mathrm{N}_{2}$ adsorption and desorption experiments were performed to investigate the surface area and porosity of the as-prepared samples. A typical type IV isotherm and type $\mathrm{H} 4$ hysteresis loop were observed for both samples (Fig. 2c). It is a typical isothermal curve of meso- and macroporous carbon materials. The specific surface area of the Sn/CNT-Agls sample $\left(71.3 \mathrm{~m}^{2} \mathrm{~g}^{-1}\right)$ increased significantly compared with the Sn/CNT sample $\left(33.7 \mathrm{~m}^{2} \mathrm{~g}^{-1}\right)$. Shown in Fig. $3 \mathrm{~b}$ is the pore size distribution curve of the $\mathrm{Sn} / \mathrm{CNT}$-Agls sample, which was obtained by the Barrett-Joyner-Halenda (BJH) method. The pore diameter of Sn/CNT-Agls is mostly mesoporous. The average pore size of $\mathrm{Sn} / \mathrm{CNT}$-Agls is $c a$. $26.5 \mathrm{~nm}$, in agreement with the SEM results (Fig. 2a and b). Consequently, it is expected that the 3D hierarchical porous structure will make the mass transfer process easier.

X-ray diffraction (XRD) and X-ray photoelectron spectroscopy (XPS) were performed to investigate the composition of the catalyst. The XRD result (Fig. $\mathrm{S} 2 \dagger$ ) confirmed the formation of metallic state Sn (PDF\#86-2264) after calcination reduction under an $\mathrm{H}_{2}$ atmosphere. Additionally, there is no obvious difference between the diffraction peaks of the Sn/CNT and Sn/ CNT-Agls samples. This indicates that the nucleation and growth of Sn particles are not affected by the physical crosslinking and freeze drying processes. The XPS survey spectrum (Fig. S5a $\dagger$ ) demonstrates that the elements $\mathrm{Sn}, \mathrm{O}, \mathrm{C}$ and $\mathrm{N}$ are present on the surface of the Sn/CNT-Agls, and the high resolution Sn 3d XPS spectrum (Fig. S6†) reveals that a native $\mathrm{SnO}_{x}$ layer exists on the surface of the Sn particles, which is attributed to the exposure to an ambient atmosphere (related discussion in the ESI $\dagger$ ). In addition, no significant differences are observed between the Sn/CNT-Agls and Sn/CNT (Fig. S5a and b $\dagger$ ).

Cyclic voltammograms (CVs) were obtained to examine the electrochemical activity of Sn/CNT-Agls/CC and Sn/CNT/CC electrodes in $0.5 \mathrm{M} \mathrm{KHCO}_{3}$ saturated with $\mathrm{CO}_{2}$ or $\mathrm{N}_{2}$ (Fig. 3a). The oxidation peaks at $c a .-0.16 \mathrm{~V}$ and $0.14 \mathrm{~V}$ correspond to the process in which $\mathrm{Sn}^{0}$ is oxidized to $\mathrm{Sn}^{2+}$ and $\mathrm{Sn}^{4+}$, respectively. The reduction peak at $c a .0 .26 \mathrm{~V}$ is assigned to the process in

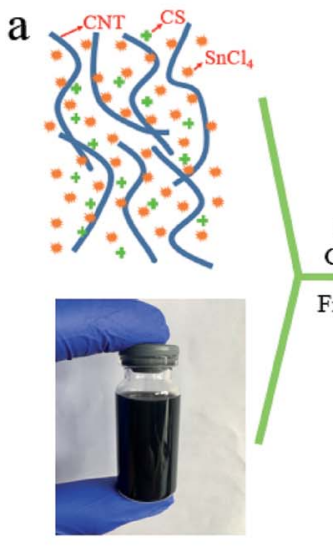

Hybrid Suspension

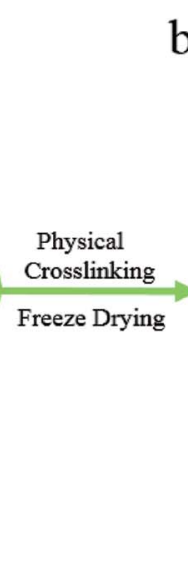

$\mathrm{Sn}(\mathrm{OH})_{\mathrm{x}} / \mathrm{CNT}$-Aerogel

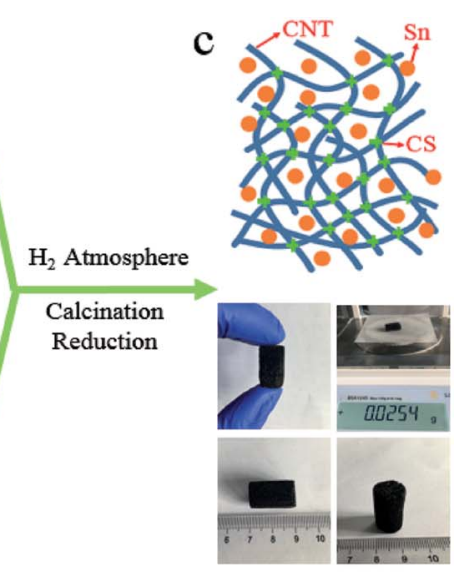

Sn/CNT-Aerogel

Fig. 1 The fabrication process of the 3D Sn/CNT-Agls catalyst. (a) Stable hybrid suspension of CNTs, CS and $\mathrm{SnCl} 4$. (b) Sn(OH) $/ \mathrm{CNT}$-Agls obtained by physical crosslinking self-assembly and freeze drying. (c) Sn/CNT-Agls obtained after calcination reduction under an $\mathrm{H}_{2}$ atmosphere. 

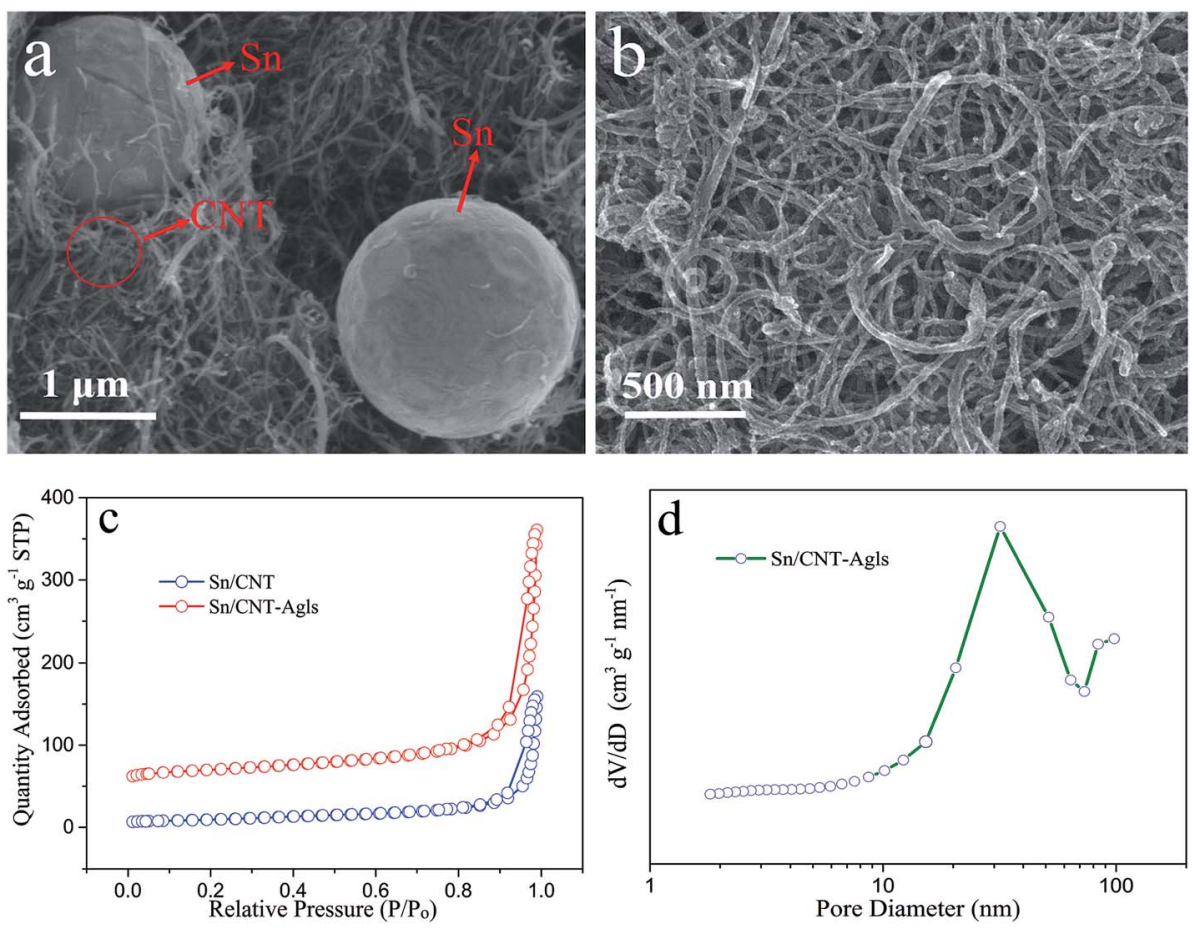

Fig. 2 Typical SEM images of Sn/CNT-Agls (a and b). Nitrogen adsorption-desorption isotherms of Sn/CNT and Sn/CNT-Agls (c) and the corresponding BJH pore size distribution curve of Sn/CNT-Agls (d).

which $\mathrm{Sn}^{4+}$ is reduced to $\mathrm{Sn}^{0}{ }^{28}$ For the Sn/CNT-Agls/CC electrode, the onset reduction potential under $\mathrm{N}_{2}(-0.74 \mathrm{~V})$ is more negative than that under $\mathrm{CO}_{2}(-0.61 \mathrm{~V})$. Moreover, a sharp increase of current density under both $\mathrm{N}_{2}$ and $\mathrm{CO}_{2}$ can be observed at more negative potentials, and the current density under $\mathrm{N}_{2}$ is lower than that under $\mathrm{CO}_{2}$. The sharp increase of the current density under $\mathrm{N}_{2}$ should be caused by the electrolysis of water $\left(\mathrm{H}_{2}\right.$ evolution reaction) and that under $\mathrm{CO}_{2}$ is attributed to both the electrolysis of water and the reduction of $\mathrm{CO}_{2}{ }^{29}$ Obviously, the $\mathrm{Sn} / \mathrm{CNT}$-Agls/CC electrode exhibits a higher electrochemical activity than the Sn/CNT/CC electrode under $\mathrm{CO}_{2}$. It can be seen that the current density of the $\mathrm{Sn} /$ CNT-Agls/CC electrode is three times higher than that of the Sn/CNT/CC electrode, which should be caused by the high specific surface area, multiple catalytically active sites and excellent conductive network structure of the Sn/CNT-Agls/CC electrode. Together, these results indicate that the Sn/CNTAgls/CC electrode exhibits excellent electrocatalytic activities toward $\mathrm{CO}_{2}$ reduction. Furthermore, the control mechanism of electrochemical reaction on the Sn/CNT-Agls/CC electrode was investigated by using the cyclic voltammograms at different sweep rates (Fig. S7 $\dagger$ ).

Electrochemical impedance spectroscopy (EIS) was performed in $\mathrm{CO}_{2}$ saturated $0.5 \mathrm{M} \mathrm{KHCO}_{3}$ electrolyte solution to investigate the kinetic behaviors on the $\mathrm{Sn} / \mathrm{CNT} / \mathrm{CC}$ and $\mathrm{Sn} /$ CNT-Agls/CC electrodes. It has been known from the CV results that the onset reduction potential toward $\mathrm{CO}_{2}$ reduction is around $-0.61 \mathrm{~V}$. Consequently, $-0.61 \mathrm{~V}$ was selected as the
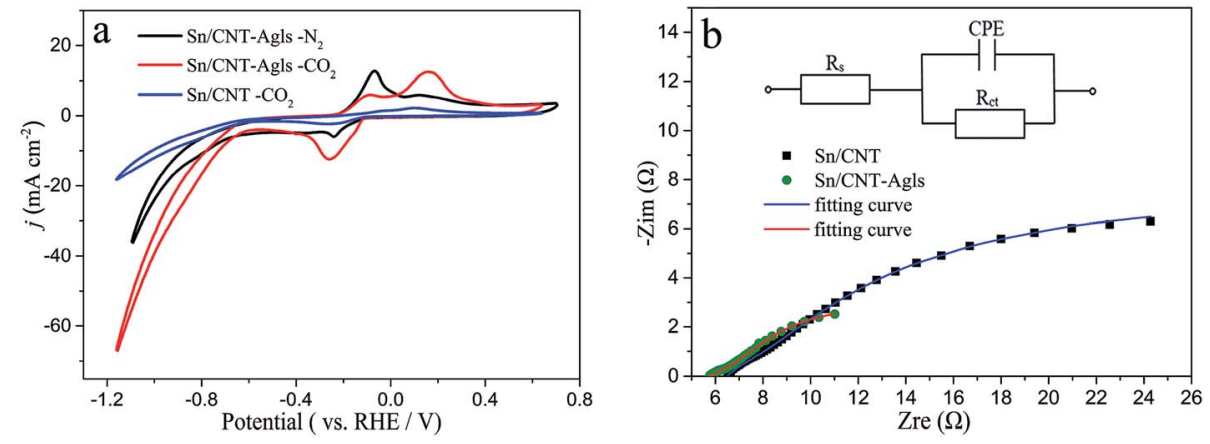

Fig. $3 \mathrm{CVs}$ of $\mathrm{Sn} / \mathrm{CNT}$-Agls/CC and $\mathrm{Sn} / \mathrm{CNT} / \mathrm{CC}$ electrodes in $0.5 \mathrm{M} \mathrm{KHCO}_{3}$ saturated with $\mathrm{CO}_{2}$ or $\mathrm{N}_{2}$ at a sweep rate of $50 \mathrm{mV} \mathrm{s}{ }^{-1}$ (a). The Nyquist diagrams and fitting curves of the Sn/CNT/CC and Sn/CNT-Agls/CC electrodes in $0.5 \mathrm{M} \mathrm{KHCO}_{3}$ and the inset is the fitting equivalent circuit that corresponds to the Nyquist diagrams (b). 
test potential of EIS in this experiment. The Nyquist diagrams and the fitting equivalent circuit are shown in Fig. $4 \mathrm{~b}$ and the fitting values of the electronic components in the equivalent circuit are shown in Table 1 . The $R_{\mathrm{s}}$ represents the body impedance of the substrate electrode, the catalyst and the impedance caused by contact with the electrolyte solution, corresponding to the first intersection with the real axis of the Nyquist plots in the high frequency region. Because the same substrate electrode (carbon cloth) and electrolyte solution $\left(0.5 \mathrm{M} \mathrm{KHCO}_{3}\right)$ are used, the magnitude of the $R_{\mathrm{S}}$ value indicates the change in the body impedance of the as-prepared electrocatalysts. The $R_{\text {ct }}$ represents the charge transfer resistance, which indicates the difficulty of kinetic reaction in the electrocatalytic process. It can be seen that the $R_{\mathrm{s}}$ value of the Sn/CNTAgls/CC electrode is smaller than that of the Sn/CNT/CC electrode. This result demonstrates that the electrical conductivity of the Sn/CNT-Agls/CC electrode improved compared with the $\mathrm{Sn} / \mathrm{CNT} / \mathrm{CC}$ electrode. Furthermore, the $R_{\mathrm{ct}}$ value of the Sn/CNTAgls/CC electrode is smaller compared with the Sn/CNT electrode. This is attributed to the superior conductivity and excellent conductive network structure of the Sn/CNT-Agls. The interfacial electron transfer rate is improved and the difficulty of kinetic reaction is reduced in the process of $\mathrm{CO}_{2}$ reduction after being crosslinked into the 3D structured Sn/CNT-Agls.

The effect of electrolysis potential on the current density and faradaic efficiency for formate production on the Sn/CNT-Agls/ CC electrode was investigated by the controlled potential electrolysis method, and the results are shown in Fig. 4a. In this experiment, no $\mathrm{CO}$ and $\mathrm{CH}_{4}$ were detected and the gas product was only $\mathrm{H}_{2}$ for the Sn/CNT-Agls/CC and Sn/CNT/CC electrodes. Formate and $\mathrm{H}_{2}$ together accounted for $>99 \%$ of the reduction products in the $\mathrm{KHCO}_{3} / \mathrm{CO}_{2}$ electrolyte. In addition, no formate was detected for the pure CNTs/CC and CNT-Agls/CC electrodes (Fig. S8†). As previously reported for the Sn electrodes, the current density increases with the electrolysis potential. ${ }^{\mathbf{1 9 2 1}}$ The faradaic efficiency for producing formate increases from -0.56 to $-0.96 \mathrm{~V}$ and then decreases at more negative electrolysis potentials. The maximum value $(82.7 \%)$ is obtained with a current density of $26.7 \mathrm{~mA} \mathrm{~cm} \mathrm{~cm}^{-2}$ at a moderate applied potential of $-0.96 \mathrm{~V}$. Since the $\mathrm{CO}_{2} / \mathrm{HCOO}^{-}$equilibrium potential is $-0.2 \mathrm{~V} v s$. the $\mathrm{RHE}^{30}-0.96 \mathrm{~V}$ corresponds to $0.76 \mathrm{~V}$ overpotential for $\mathrm{HCOO}^{-}$production. Additionally, at a low
Table 1 The fitting results of the EIS equivalent circuit

\begin{tabular}{lcc}
\hline Electrode & $R_{\mathrm{s}}$ & $R_{\mathrm{ct}}$ \\
\hline Sn/CNT & 6.5 & 40.1 \\
Sn/CNT-Agls & 5.7 & 12.6 \\
\hline
\end{tabular}

overpotential of $0.36 \mathrm{~V}(-0.56 \mathrm{~V} v s$. the RHE), formate can still be detected on the Sn/CNT-Agls/CC electrode with a faradaic efficiency of $20.9 \%$. Strikingly, a large current density of $32.9 \mathrm{~mA}$ $\mathrm{cm}^{-2}$ (the current density based on the Sn mass loading is $20.6 \mathrm{~A} \mathrm{~g}^{-1}$ ) with a relatively high faradaic efficiency at $-1.16 \mathrm{~V}$ was achieved on the Sn/CNT-Agls/CC electrode, which is the maximum of all reported values of Sn electrodes and even larger than those of most $\mathrm{Sn}$ gas diffusion electrodes in aqueous solution (Table S1†). The faradaic efficiency decreases as the electrolysis potential increases further, which is attributed to the enhancement of $\mathrm{H}_{2}$ evolution, which competes with $\mathrm{CO}_{2}$ reduction to formate under these conditions. ${ }^{31,32}$ The above results indicate that the appropriate potential for producing formate on the Sn/CNT-Agls/CC electrode could be around $-0.96 \mathrm{~V}$ and the potential dependence of formate selectivity is consistent with previously reported results. ${ }^{33}$ Further experiments were carried out on the Sn/CNT/CC and Sn/CNT-Agls/CC electrodes at $-0.96 \mathrm{~V}$ to compare their electrocatalytic activities for the electrochemical reduction of $\mathrm{CO}_{2}$ to formate, and the results are shown in Fig. 4b. The faradaic efficiency of the $\mathrm{Sn} /$ CNT-Agls/CC electrode is 1.5-fold higher than that of the $\mathrm{Sn}$ / $\mathrm{CNT} / \mathrm{CC}$ electrode. Moreover, the current density and production rate on the Sn/CNT-Agls/CC electrode are far greater than those on the Sn/CNT/CC electrode. The above results reveal that the electrocatalytic activity of $\mathrm{CO}_{2}$ reduction on the Sn/CNTAgls/CC electrode is far superior to that on the $\mathrm{Sn} / \mathrm{CNT} / \mathrm{CC}$ electrode. The excellent electrocatalytic activity on the $\mathrm{Sn} /$ CNT-Agls/CC electrode can be attributed to the following three reasons. Firstly, the 3D hierarchical porous structured $\mathrm{Sn} /$ CNT-Agls shows a higher specific surface area (Fig. 2c and d) than the Sn/CNT composites. Secondly, a 3D hierarchical porous structure of Sn/CNT-Agls (Fig. 2a-d) makes all the catalytically active sites more easily accessible to the reactants, resulting in a greatly accelerated mass transfer process. Consequently, the difficulty of kinetic reaction toward $\mathrm{CO}_{2}$
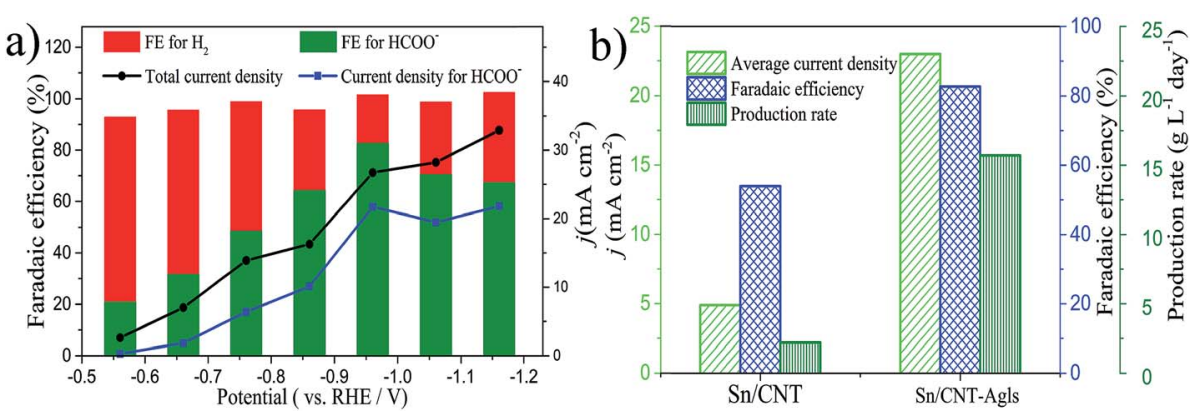

Fig. 4 Variations of the average current density and faradaic efficiency with the electrolysis potential on the Sn/CNT-Agls/CC electrode (a). Comparison of the average current density, Faraday efficiency and production rate of formate on Sn/CNT/CC and Sn/CNT-Agls/CC electrodes at $-0.96 \mathrm{~V}(\mathrm{~b})$. 
a)

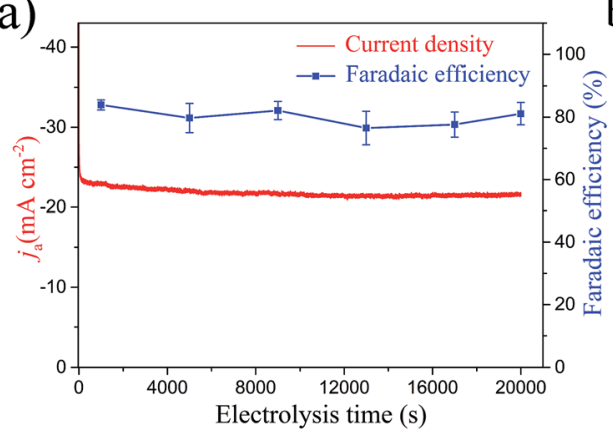

b)

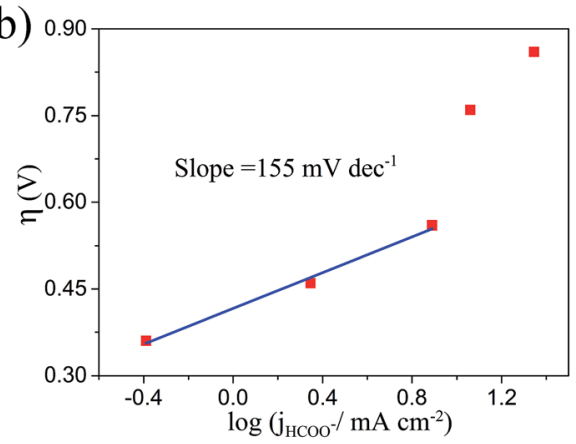

Fig. 5 Variations of the current density and faradaic efficiency for formate production with the electrolysis time on the Sn/CNT-Agls/CC electrode at $-0.96 \mathrm{~V}$ (a). Formate partial current density Tafel plots of the Sn/CNT-Agls/CC electrode (b).

reduction had been reduced greatly. Thirdly, the interfacial electron transfer rate had been greatly accelerated (Fig. 3b) due to the good conductivity of the Sn/CNT-Agls (Fig. 3b) and the excellent 3D conductive network structure. Together, it can be concluded that the electrocatalytic activity of Sn/CNT for producing formate is greatly enhanced after being crosslinked into the Sn/CNT-Agls.

To investigate the stability of the Sn/CNT-Agls/CC electrode during the process of $\mathrm{CO}_{2}$ reduction, a long-term constant potential electrolysis experiment was carried out at an applied electrolysis potential of $-0.96 \mathrm{~V}$ for $20000 \mathrm{~s}$ (Fig. 5a). The faradaic efficiency for formate production was analyzed every $4000 \mathrm{~s}$ in the process of the electrolysis experiment. It can be seen that the Sn/CNT-Agls/CC electrode exhibits excellent stability during the long-term electrolysis experiment. A slight decrease of current density was observed during the long term process of $\mathrm{CO}_{2}$ reduction and a faradaic efficiency of $c a$. $82 \%$ was maintained even after continuous electrolysis for $20000 \mathrm{~s}$. Furthermore, the morphology of Sn particles did not change significantly during electrolysis (Fig. S2 $\dagger$ ). Naturally, it is feasible to produce formate continuously with a high stability and faradaic efficiency on the Sn/CNT-Agls/CC electrode.

According to previously reported results, a possible mechanism of electrochemical reduction of $\mathrm{CO}_{2}$ for producing formate on $\mathrm{Sn}$ electrodes is illustrated as the following 5 equations. ${ }^{18,19}$

$$
\begin{gathered}
\mathrm{CO}_{2} \text { (solution) } \rightarrow \mathrm{CO}_{2} \text { (ads) } \\
\mathrm{CO}_{2} \text { (ads) }+\mathrm{e}^{-} \rightarrow \mathrm{CO}_{2}^{--} \text {(ads) } \\
\mathrm{CO}_{2}^{\cdot-} \text { (ads) }+\mathrm{HCO}_{3}^{-}+\mathrm{e}^{-} \rightarrow \mathrm{HCOO}^{-} \text {(ads) }+\mathrm{CO}_{3}^{2-} \\
\mathrm{HCOO}^{-} \text {(ads) } \rightarrow \mathrm{HCOO}^{-} \text {(solution) } \\
\mathrm{CO}_{2}+\mathrm{CO}_{3}^{2-}+\mathrm{H}_{2} \mathrm{O} \rightarrow 2 \mathrm{HCO}_{3}^{-}
\end{gathered}
$$

In order to determine the chemical rate-determining step (RDS) in the reaction mechanism, the Tafel analysis for the production of formate was performed on the Sn/CNT-Agls/CC electrode and the result is shown in Fig. 5b. A slope of $155 \mathrm{mV} \mathrm{dec}^{-1}$ in the low overpotential region is observed on the
Sn/CNT-Agls/CC electrode. The value is close to the slope of $118 \mathrm{mV} \mathrm{dec}^{-1}$ (120 $\mathrm{mV} \mathrm{dec}^{-1}$ was reported by S. Kapusta), which suggests that the RDS was the initial single-electron transfer to hydrated $\mathrm{CO}_{2}$ to form a surface-adsorbed $\mathrm{CO}_{2}{ }^{--}$intermediate. ${ }^{18,34,35}$ Therefore, a hydrated $\mathrm{CO}_{2}$ molecule obtains an electron to form a surface adsorbed $\mathrm{CO}_{2}{ }^{--}$intermediate, which is the rate limiting chemical step (eqn (2)) in this experiment.

In summary, a 3D hierarchical porous structured Sn/CNTAgls electrocatalyst for efficient electrochemical reduction of $\mathrm{CO}_{2}$ to formate has been fabricated successfully via a simple three-step process. Because of the $3 \mathrm{D}$ hierarchical porous structure, the high specific surface area and the superior conductive network structure of Sn/CNT-Agls, the as-prepared electrode exhibits excellent electrocatalytic activity toward $\mathrm{CO}_{2}$ reduction, including a large current density, a high faradaic efficiency and production rate, a good stability and a moderate overpotential. In addition, it will be interesting and meaningful if suitable synthetic conditions can be found to control the size of the Sn particles. Our research has opened up a new way for the preparation of high-performance 3D porous structured catalyst materials toward $\mathrm{CO}_{2}$ reduction. It can be expected that many other metals/carbon-based 3D materials as efficient electrodes for $\mathrm{CO}_{2}$ reduction could be prepared via this simple method.

\section{Conflicts of interest}

There are no conflicts to declare.

\section{Acknowledgements}

This work was supported by the CAS Hundred Talent Program and the National Natural Science Foundation of China (Grant No. 21676288).

\section{References}

1 A. Otto, T. Grube, S. Schiebahn and D. Stolten, Energy Environ. Sci., 2015, 8, 3283-3297.

2 T. N. Huan, E. S. Andreiadis, J. Heidkamp, P. Simon, E. Derat, S. Cobo, G. Royal, A. Bergmann, P. Strasser and H. Dau, J. Mater. Chem. A, 2015, 3, 3901-3907. 
3 S. Rasul, D. H. Anjum, A. Jedidi, Y. Minenkov, L. Cavallo and K. Takanabe, Angew. Chem., Int. Ed., 2015, 127, 2174-2178.

4 D. Gao, H. Zhou, J. Wang, S. Miao, F. Yang, G. Wang, J. Wang and X. Bao, J. Am. Chem. Soc., 2015, 137, 4288-4291.

5 A. A. Peterson, F. Abild-Pedersen, F. Studt, J. Rossmeisl and J. K. Nørskov, Energy Environ. Sci., 2010, 3, 1311-1315.

6 B. Innocent, D. Liaigre, D. Pasquier, F. Ropital, J. M. Léger and K. B. Kokoh, J. Appl. Electrochem., 2009, 39, 227.

7 H. Uslu, C. Bayat, S. Gökmen and Y. Yorulmaz, J. Chem. Eng. Data, 2008, 54, 48-53.

8 Y. Wang, J. Zhou, W. Lv, H. Fang and W. Wang, Appl. Surf. Sci., 2016, 362, 394-398.

9 N. Danilovic, R. Subbaraman, K.-C. Chang, S. H. Chang, Y. J. Kang, J. Snyder, A. P. Paulikas, D. Strmcnik, Y.-T. Kim and D. Myers, J. Phys. Chem. Lett., 2014, 5, 2474-2478.

10 J. Yang, C. Tian, L. Wang and H. Fu, J. Mater. Chem. A, 2011, 21, 3384-3390.

11 Z. M. Detweiler, J. L. White, S. L. Bernasek and A. B. Bocarsly, Langmuir, 2014, 30, 7593-7600.

12 G. S. Prakash, F. A. Viva and G. A. Olah, J. Power Sources, 2013, 223, 68-73.

13 M. Alvarez-Guerra, S. Quintanilla and A. Irabien, Chem. Eng. J., 2012, 207, 278-284.

14 H.-Z. Zhao, Y. Zhang, Y.-Y. Chang and Z.-S. Li, J. Power Sources, 2012, 217, 59-64.

15 A. Del Castillo, M. Alvarez-Guerra, J. Solla-Gullón, A. Sáez, V. Montiel and A. Irabien, Appl. Energy, 2015, 157, 165-173.

16 Q. Wang, H. Dong and H. Yu, RSC Adv., 2014, 4, 5997059976.

17 Q. Wang, H. Dong and H. Yu, J. Power Sources, 2014, 271, 278-284.

18 C. Zhao and J. Wang, Chem. Eng. J., 2016, 293, 161-170.
19 S. Zhang, P. Kang and T. J. Meyer, J. Am. Chem. Soc., 2014, 136, 1734-1737.

20 M. F. Baruch, J. E. Pander III, J. L. White and A. B. Bocarsly, ACS Catal., 2015, 5, 3148-3156.

21 W. Lv, R. Zhang, P. Gao and L. Lei, J. Power Sources, 2014, 253, 276-281.

22 R. L. Machunda, H. Ju and J. Lee, Curr. Appl. Phys., 2011, 11, 986-988.

23 A. S. Agarwal, Y. Zhai, D. Hill and N. Sridhar, ChemSusChem, 2011, 4, 1301-1310.

24 Y. Hori, H. Wakebe, T. Tsukamoto and O. Koga, Electrochim. Acta, 1994, 39, 1833-1839.

25 Y. Chen and M. W. Kanan, J. Am. Chem. Soc., 2012, 134, 1986-1989.

26 R. R. Kohlmeyer, M. Lor, J. Deng, H. Liu and J. Chen, Carbon, 2011, 49, 2352-2361.

27 M. B. Bryning, D. E. Milkie, M. F. Islam, L. A. Hough, J. M. Kikkawa and A. G. Yodh, Adv. Mater., 2007, 19, 661-664.

28 S. D. Kapusta and N. Hackerman, Electrochim. Acta, 1980, 25, 1625-1639.

29 R. Zhang, W. Lv and L. Lei, Appl. Surf. Sci., 2015, 356, 24-29.

30 C. W. Li and M. W. Kanan, J. Am. Chem. Soc., 2012, 134, 7231.

31 W. Lv, R. Zhang, P. Gao and L. Lei, J. Power Sources, 2014, 253, 276-281.

32 S. Zhang, P. Kang and T. J. Meyer, J. Am. Chem. Soc., 2014, 136, 1734.

33 F. Li, L. Chen, G. P. Knowles, D. R. MacFarlane and J. Zhang, Angew. Chem., Int. Ed., 2017, 129, 520-524.

34 S. Kapusta and N. Hackerman, J. Electrochem. Soc., 1983, 130, 607-613.

35 D. H. Won, C. H. Choi, J. Chung, M. W. Chung, E. H. Kim and S. I. Woo, ChemSusChem, 2015, 8, 3092-3098. 